\title{
Compton scattering in noncommutative space-time at the Next Linear Collider
}

\author{
Prakash Mathews* \\ Instituto de Fisica Teorica, Universidade Estadual Paulista, Rua Pamplona 145, 01405-900, Sao Paulo, SP, Brazil
}

(Received 4 December 2000; published 5 March 2001)

\begin{abstract}
We study Compton scattering in the noncommutative (NC) counterpart of QED. Interactions in NC QED have momentum dependent phase factors and the gauge fields have Yang-Mills type couplings; this modifies the cross sections and they are different from the commuting standard model. Collider signals of noncommutative space-time are studied at the Next Linear Collider (NLC) operating in the $e \gamma$ mode. Results for different polarized cases are presented and it is shown that the Compton process can probe the noncommutative scale in the range of 1-2.5 TeV for typical proposed NLC energies.
\end{abstract}

DOI: 10.1103/PhysRevD.63.075007

PACS number(s): 12.60.-i, 13.40.-f

\section{INTRODUCTION}

The idea that space-time could be noncommutative (NC) dates back to the work of Snyder [1] in 1947. Noncommutative field theory (NCFT) has been of recent interest following the realization in string theory that $\mathrm{NC}$ spaces come about in a specific low energy limit of D-brane dynamics in the presence of a certain $U(1)$ constant background magnetic field [2]. Prior to this, noncommutative geometry and the field theoretical construction on it was developed purely in a general mathematical framework [3]. The noncommutativity of such a space can be characterized by the commutator of coordinates $x^{\mu}$ :

$$
\left[x_{\mu}, x_{\nu}\right]=i \theta_{\mu \nu},
$$

where the matrix $\theta_{\mu \nu}$ is real, antisymmetric, and commutes with space-time coordinate $x^{\mu}$. The NC parameter $\theta_{\mu \nu}$ has dimensions of area and reflects the extent to which the particular coordinates noncommute. Furthermore, a NC scale $\Lambda_{\mathrm{NC}}$ can be introduced above which the coordinates are noncommuting:

$$
\left[x_{\mu}, x_{\nu}\right]=i \frac{c_{\mu \nu}}{\Lambda_{\mathrm{NC}}^{2}},
$$

where $c_{\mu \nu}$ has the same properties as $\theta_{\mu \nu}$ but the strength of noncommutativity has been absorbed in the NC scale $\Lambda_{\mathrm{NC}}$.

Various aspects of field theory on noncommutative space have been analyzed. The perturbative structure and the renormalisability of these theories have been studied [4]. One of the major out come of these studies is that the ultraviolet and infrared effects in noncommutative field theory do not decouple [5]. Hence physics at short distance can affect physics at long distance and vice versa. The causal behavior of space-space and space-time noncommutativity has been studied and shown that while space-space noncommutativity is causal and unitary, the space-time is acausal and nonunitary in NCFT, but it could be restored in string theory [6]. Apart from string theory [6], unitarity of space-time nonlocality can also be restored in super Yang-Mills theory [7].

*Email address: mathews@ift.unesp.br
Noncommutative QED (NC QED) has been shown to be one loop renormalizable $[8,9]$. The $C, P, T$ properties of $\mathrm{NC}$ QED has also been studied and is found to be $C P T$ invariant for space-space and space-time noncommutativity [10]. There has also been attempts to formulate a noncommutative Glashow-Weinberg-Salam model [11]. Hence it would be interesting to explore the possible effects of noncommutativity at the collider level.

Noncommutative effects at the colliders has been studied for the first time by Hewett et al. [12]. They have considered several $2 \rightarrow 2$ processes in $e^{+} e^{-}$collisions at the Next Linear Collider (NLC) and shown that NC scale of the order of a $\mathrm{TeV}$ can be probed at the NLC. Though the NC scale $\Lambda_{\mathrm{NC}}$ could be the Planck or the grand unified theory (GUT) scale it was argued in the context of the recent work on possible $\mathrm{TeV}$ scale quantum gravity $[17,18]$ that one could as well consider the NC scale $\Lambda_{\mathrm{NC}}$ to be not too far from the TeV scale [12]. They have considered the Moller, Bhabha, and annihilation processes in the $e^{+} e^{-}$mode and the $\gamma \gamma \rightarrow \gamma \gamma$ process in the $\gamma \gamma$ mode of NLC. The $95 \%$ lower bound on the $\Lambda_{\mathrm{NC}}$ for the various process obtained are (a) $1700 \mathrm{GeV}$ for Moller, (b) $1050 \mathrm{GeV}$ for Bhabha, (c) in the range 740$840 \mathrm{GeV}$ for annihilation, and (e) for $\gamma \gamma \rightarrow \gamma \gamma$ the spacetime NC yields $700-800 \mathrm{GeV}$ while space-space $\mathrm{NC}$ gives $500 \mathrm{GeV}$ [12]. Other phenomenological aspects of noncommutativity has been studied [13-15] and bounds on the $\theta$ parameter obtained for high- and low-energy limits.

The NLC is planned to operate in $e^{+} e^{-}, \gamma \gamma$, and $e \gamma$ modes. At high energy and luminosity, the $e^{+} e^{-}$collider can yield a $\gamma$ beam of comparable energy and luminosity using the laser back scattering technique [19]. The NLC is an ideal testing ground of the standard model (SM) and a very effective probe of possible physics that may lie beyond the SM. The experiment at the NLC also provides a great degree of precision because of the relatively clean initial states and the degree of precision can be enhanced by using the polarization of the initial states. Hence we have considered the possibility of testing the NC effects at the NLC in the $e \gamma$ mode by studying the Compton scattering.

In Sec. II we introduce the basics of NC QED. In Sec. III we present the cross section for Compton scattering in $\mathrm{NC}$ space. The numerical results and possible reach of the NLC is discussed in Sec. IV. Finally we summarize our results in Sec. V. 


\section{NC QED}

The one loop UV divergent structure of U(1) Yang-Mills theory on noncommutative $R^{4}$ has been analyzed [8]. The mathematical structure of the quantum field theory over a noncommutative space is not yet well understood but by assuming that the quantum theory is defined by the generating functional of the theory, it was shown that the theory is renormalizable at the one loop level [8]. The matter fields where introduced and the perturbative aspects of NC QED have been analyzed [9]. The NC QED action is given by

$S_{\mathrm{NC} Q E D}=\int d^{d} x\left(-\frac{1}{4 g^{2}} F_{\mu \nu} * F^{\mu \nu}+\bar{\psi}^{*} \gamma^{\mu} i D_{\mu} \psi-m \bar{\psi} * \psi\right)$,

where the $*$ product is defined as

$$
A * B(x)=\left.\exp \left(\frac{i}{2} \theta_{\mu \nu} \frac{\partial}{\partial \xi^{\mu}} \frac{\partial}{\partial \eta^{\nu}}\right) A(x+\xi) B(x+\eta)\right|_{\xi, \eta=0}
$$

The noncommutativity of space modifies the algebra of functions and even in the $U(1)$ case the field strength is nonlinear and has the form $F_{\mu \nu}=\partial_{\mu} A_{\nu}-\partial_{\nu} A_{\mu}-i\left[A_{\mu}, A_{\nu}\right]_{M}$. The Moyal bracket $[A, B]_{M}$ is a commutator under the $*$ product. The covariant derivative for the matter fields is given by

$$
D_{\mu} \psi=\partial_{\mu} \psi-i A_{\mu} * \psi, \quad D_{\mu} \bar{\psi}=\partial_{\mu} \bar{\psi}+i \bar{\psi} * A_{\mu} \text {. }
$$

There exist other possible choice of covariant derivative which are related to the above by charge conjugation and are detailed in [10]. The action Eq. (3) is invariant under the NC gauge transformation

$$
\begin{aligned}
& A_{\mu}(x) \rightarrow A^{\prime}(x)=U(x) * A_{\mu}(x) * U^{-1}(x) \\
&+i U(x) * \partial_{\mu} U^{-1}(x) \\
& \psi(x) \rightarrow \psi^{\prime}(x)=U(x) * \psi(x) \\
& \bar{\psi}(x) \rightarrow \bar{\psi}^{\prime}(x)=\bar{\psi}(x) * U(x)^{-1}
\end{aligned}
$$

where $U(x)=\exp [i \alpha(x)]_{*}$ is defined as an infinite series of the scalar function $\alpha(x)$ under the $*$ product and $U(x)^{-1}$ is its inverse. Note that due to the $*$ product algebra of the $\mathrm{NC}$ space, the Lagrangian is no longer invariant under the NC gauge transformation but the action is. Hence this gives the basic ingredients needed to derive the Feynman rules [9]. Each of the interaction vertex picks up a phase factor which depends on the momentum and even in the $U(1)$ case, $A_{\mu}$ couples to itself and there exist three and four point photon vertices. The propagators are the same as the commuting theory. Due to noncommutativity, the theory is manifestly nonlocal in the noncommuting coordinates and this leads to violation of Lorentz invariance.

\section{COMPTON SCATTERING IN NC QED}

The Feynman diagrams that contributes to the tree level process $\gamma\left(k_{1}\right) e\left(p_{1}\right) \rightarrow \gamma\left(k_{2}\right) e\left(p_{2}\right)$ in the NC QED has the usual $s$ - and $u$-channel diagrams and in addition there is also a $t$-channel diagram coming from the triple photon vertex. The amplitude is given by

$$
\begin{aligned}
\mathcal{M}= & -i g^{2} \exp \left(\frac{i}{2} p_{1} \wedge p_{2}\right) \epsilon_{1 \mu}\left(k_{1}\right) \epsilon_{2 \nu}^{*}\left(k_{2}\right) \\
& \times\left[\left(\frac{1}{\hat{s}} \bar{u}\left(p_{2}\right) \gamma_{\nu}\left(p_{1}+k_{1}\right) \gamma_{\mu} u\left(p_{1}\right)\right.\right. \\
& \left.+\frac{V^{\mu \nu \rho}}{\hat{t}} \bar{u}\left(p_{2}\right) \gamma_{\rho} u\left(p_{1}\right)\right) \exp \left(\frac{i}{2} p_{12} \wedge k_{2}\right) \\
& +\left(\frac{1}{\hat{u}} \bar{u}\left(p_{2}\right) \gamma_{\mu}\left(p_{1}-k_{2}\right) \gamma_{\nu} u\left(p_{1}\right)\right. \\
& \left.\left.-\frac{V^{\mu \nu \rho}}{\hat{t}} \bar{u}\left(p_{2}\right) \gamma_{\rho} u\left(p_{1}\right)\right) \exp \left(\frac{-i}{2} p_{12} \wedge k_{2}\right)\right],
\end{aligned}
$$

where $g$ is the NC coupling, $p_{12}=p_{1}-p_{2}$, the caret on the Mandelstam variables corresponds to the $e \gamma$ subprocess and the three photon vertex function $V$ is given by

$$
V_{\mu \nu \rho}=g_{\mu \rho}\left(2 k_{1}-k_{2}\right)_{\nu}+g_{\nu \rho}\left(2 k_{2}-k_{1}\right)_{\mu}-g_{\mu \nu}\left(k_{1}+k_{2}\right)_{\rho} \text {. }
$$

The $\wedge$ product is defined as

$$
p_{1} \wedge p_{2}=\theta_{\mu \nu} p_{1}^{\mu} p_{2}^{\nu}=\frac{c_{\mu \nu}}{\Lambda_{N C}^{2}} p_{1}^{\mu} p_{2}^{\nu}
$$

The NC effects appear as the phase factors. In the commuting limit $\theta \rightarrow 0$ the SM diagrams are recovered. Because of the triple photon NC contribution, care should be taken to check the Ward identity and to retain only the physical photon degrees of freedom. The NC effects at the cross section could arise from the interference of diagrams which could pick up a phase or from the explicit three and four photon vertex diagrams.

At the NLC, $\gamma$ beam can be obtained from laser back scattering and hence has a distribution in energy and helicity of the parent electron and laser beam. The differential cross section for the Compton scattering at the NLC is given by

$$
\begin{aligned}
\frac{d \sigma}{d \Omega}= & \alpha^{2} \int d x \frac{f(x)}{x s}\left(\frac{1+P_{e_{2}} \xi_{2}(x)}{2}\left|\mathcal{M}_{++}\right|^{2}\right. \\
& \left.+\frac{1-P_{e_{2}} \xi_{2}(x)}{2}\left|\mathcal{M}_{+-}\right|^{2}\right),
\end{aligned}
$$

where $\alpha=g^{2} / 4 \pi, x$ is the fraction of the parent electron energy carried by the photon, $s$ corresponds to the c.m.s. lab frame of the $e^{+} e^{-}$pair, and $P_{e_{2}}$ is the beam polarization of the electron beam. The photon number density $f(x)$ and the 
Stokes parameter $\xi_{2}(x)$ are functions of $x$, the parent electron and laser beam polarization. The details are given in the Appendix. It has been suggested in Ref. [15] to study the low-energy Thomson limit of the Compton scattering in NC case to find the physical value of NC coupling as in the QED case [16]. Whether this property of Thomson limit holds for the NC case is being investigated [15].

The matrix element square $\left|M_{i j}\right|^{2}$ corresponds to the subprocess $\gamma(i) e(j) \rightarrow \gamma e$, where $i, j$ denotes the respective initial helicities while the final helicities are summed over. The polarized $\left|M_{i j}\right|^{2}$ are given by

$$
\begin{aligned}
& \left|M_{++}\right|^{2}=-\frac{\hat{s}}{\hat{u}}+4 \frac{\hat{s}^{2}}{\hat{t}^{2}} \sin ^{2} \Delta_{C}, \\
& \left|M_{+-}\right|^{2}=-\frac{\hat{u}}{\hat{s}}+4 \frac{\hat{u}^{2}}{\hat{t}^{2}} \sin ^{2} \Delta_{C},
\end{aligned}
$$

where the noncommuting phase is

$$
\Delta_{C}=\frac{1}{2} p_{12} \wedge k_{2} .
$$

The NC effects for the Compton scattering reside in the even function $\sin ^{2} \Delta_{C}$ and taking the commuting limit $\Delta_{C} \rightarrow 0$ one recovers the SM form. The commuting limit seems possible at the tree level but in the far infrared the physics might look different due to loop effects and ultraviolet-infrared mixing $[8,9]$. To perform the phenomenology we assume the terms independent of the NC scale as the SM contribution and study the effects induced by the noncommutativity.

The NC Compton phase in the above equation is an out come of a combination of phase effects arising from the $t$-channel triple photon vertex diagram, its interference with the $s$ - and $u$-channel diagrams and the interference between the $s$ - and $u$-channel diagrams. The polar angle dependence of the above equation is different for the commuting SM and can be suitably exploited to distinguish the NC QED contribution. The cross section Eq. (10) is a function of the polarization of the electron, the parent electron and laser beams. The polarization can be used as a useful tool for the analysis.

To evaluate $\Delta_{C}$ we have to go to a specific frame and we choose the $e \gamma$ c.m. system (c.m.s.) frame

$$
\begin{aligned}
& k_{1}=\frac{\sqrt{\hat{s}}}{2}(1,0,0,1), \\
& k_{2}=\frac{\sqrt{\hat{s}}}{2}(1, \sin \theta \cos \phi, \sin \theta \sin \phi, \cos \theta), \\
& p_{1}=\frac{\sqrt{\hat{s}}}{2}(1,0,0,-1), \\
& p_{2}=\frac{\sqrt{\hat{s}}}{2}(1,-\sin \theta \cos \phi,-\sin \theta \sin \phi,-\cos \theta),
\end{aligned}
$$

where $\theta$ is the polar angle in the $e \gamma$ c.m.s. frame and the corresponding Mandelstam variables are $\hat{t}=\left(k_{1}-k_{2}\right)^{2}$ $=-\hat{s}(1-\cos \theta) / 2, \quad \hat{u}=\left(k_{1}-p_{2}\right)^{2}=-\hat{s}(1+\cos \theta) / 2$ and $\hat{s}$ $=x s$. The Compton phase factor $\Delta_{C}$ in this frame has the form

$$
\begin{aligned}
\Delta_{C}= & \frac{\hat{s}}{8 \Lambda_{N C}^{2}}\left[-c_{13} \sin \theta \cos \phi-c_{23} \sin \theta \sin \phi+c_{01} \sin \theta \cos \phi\right. \\
& \left.+c_{02} \sin \theta \sin \phi+c_{03}(-1+\cos \theta)\right] .
\end{aligned}
$$

Note that $\Delta_{C}$ gets contributions from space-space and spacetime noncommutativity. From the phase factor there is an additional polar angle dependence in the cross section and unlike the usual $2 \rightarrow 2$ process there is also an azimuthal angle dependence. The explicit form of the phase factor depends on the amount of noncommutativity of the particular noncommuting coordinates, i.e., the magnitude of the components of $c_{\mu \nu}$. For the numerical analysis we choose a few specific cases described in the next section.

\section{NUMERICAL RESULTS}

Before we present our numerical analysis it is important to point out that the phase factor $\Delta_{C}$ depends on the choice of frame and hence the cross section is not Lorentz invariant. But nonlocality and violation of Lorentz invariance are manifestation of NC spaces and if the space is indeed noncommuting above some scale $\Lambda_{\mathrm{NC}}$ the effects of these violation could be observed close to $\Lambda_{\mathrm{NC}}$. This could for instance be an azimuthal angle dependence in a $2 \rightarrow 2$ process. As was pointed out in Ref. [12], two experiments could still compare results by converting to a common frame of reference which could be some slowly varying astronomical coordinate system.

To perform the analysis we consider two cases viz. (I) $c_{0 i}=0$ and $c_{i j} \neq 0$ (space-space NC) and (II) $c_{0 i} \neq 0$ and $c_{i j}$ $=0$ (space-time NC). Further assuming the components of $c_{\mu \nu}$ to be of order of unity

$$
\begin{aligned}
\Delta_{C}^{\mathrm{I}}= & -\frac{\hat{s}}{8 \Lambda_{\mathrm{NC}}^{2}} \sin \theta \cos (\phi-\beta) ; \quad c_{0 i}=0, \quad c_{i j} \neq 0, \\
\Delta_{C}^{\mathrm{II}}= & \frac{\hat{s}}{8 \Lambda_{\mathrm{NC}}^{2}}[-\cos \alpha+\cos \alpha \cos \theta+\sin \alpha \sin \theta \\
& \times \cos (\phi-\beta)] ; \quad c_{0 i} \neq 0, \quad c_{i j}=0,
\end{aligned}
$$

where in case I the noncommutativity is parametrized as $c_{13}=\cos \beta, c_{23}=\sin \beta$ and in case II, $c_{01}=\sin \alpha \cos \beta, c_{02}$ $=\sin \alpha \sin \beta$, and $c_{03}=\cos \alpha$. The angle $\beta$ fixes the origin from where the azimuthal angle $\phi$ is measured and is chosen to be $\beta=\pi / 2$. This chooses a specific direction and hence a violation of the Lorentz symmetry. It is instructive to look at the explicit form of the phase factor for $\beta=\pi / 2$ and for the various values of $\alpha$ 


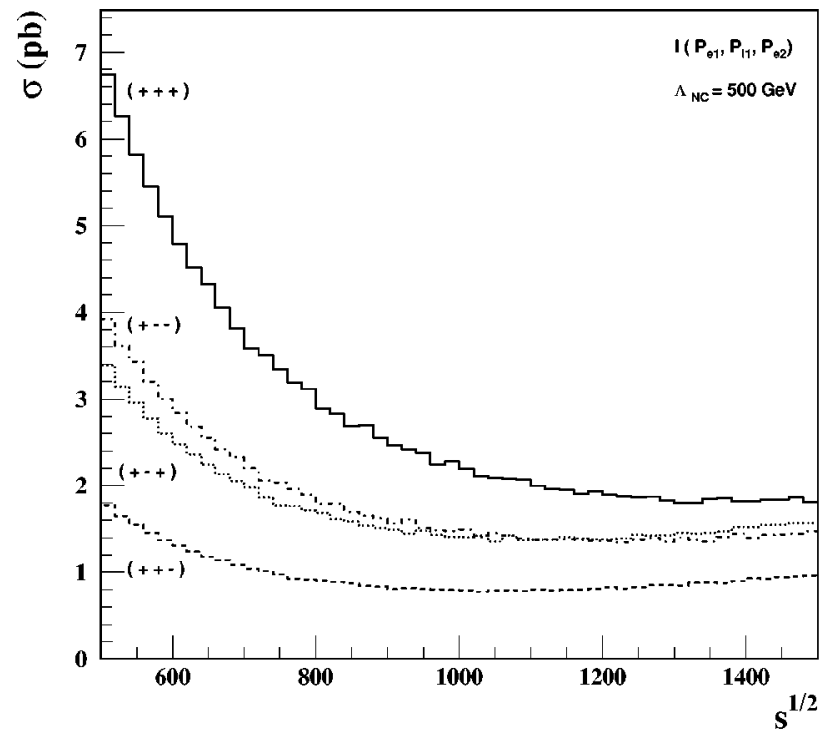

(a)

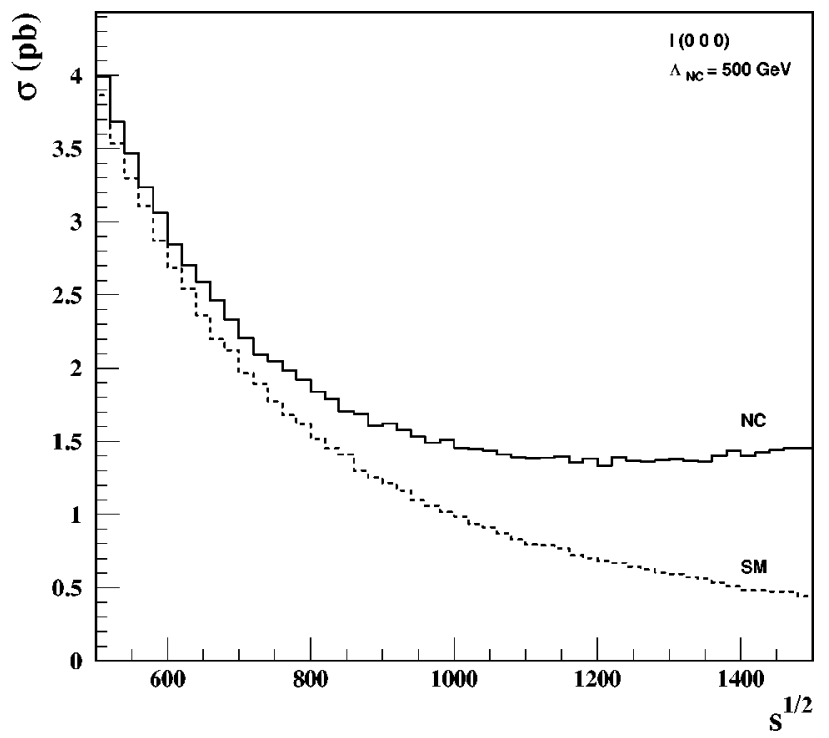

(b)

FIG. 1. (a) Total cross section as a function of $\sqrt{s}$ for various polarization states $\left(P_{e 1}, P_{l 1}, P_{e 2}\right)$. (b) Unpolarized total cross section $(0,0,0)$ as a function of $\sqrt{s}$ for the NC and SM.

$$
\begin{aligned}
\Delta_{C}^{\mathrm{I}} & =-\frac{\hat{s}}{8 \Lambda_{\mathrm{NC}}^{2}} \sin \theta \sin \phi ; c_{13}=0, c_{23}=1, \\
\left.\Delta_{C}^{\mathrm{II}}\right|_{0} & =\frac{\hat{s}}{8 \Lambda_{\mathrm{NC}}^{2}}(-1+\cos \theta) ; \quad c_{01}=0, c_{02}=0, \\
\left.\Delta_{C}^{\mathrm{II}}\right|_{\pi / 4} & =\frac{\hat{s}}{8 \sqrt{2} \Lambda_{\mathrm{NC}}^{2}}(-1+\cos \theta+\sin \theta \sin \phi) ; \\
c_{01} & =0, c_{02}=\frac{1}{\sqrt{2}}, \quad c_{03}=\frac{1}{\sqrt{2}}, \\
\left.\Delta_{C}^{\mathrm{II}}\right|_{\pi / 2} & =\frac{\hat{s}_{8}^{2}}{8 \Lambda_{\mathrm{NC}}^{2}} \sin \theta \sin \phi ; \quad c_{01}=0, c_{02}=1, c_{03}=0 .
\end{aligned}
$$

The above cases have definite meaning with respect to the constant background field responsible for the noncommutativity [2]. The space-space noncommutativity $c_{i j} \neq 0$ corresponds to a background magnetic field in the direction perpendicular to $i j$ while the space-time noncommutativity $c_{0 i}$ $\neq 0$ corresponds to a background electric field in the direction characterized by an angle $\alpha$ with respect to the beam direction. Cases I and IIc $(\alpha=\pi / 2)$ have the same form at the cross section level. Case IIa does not have an azimuthal angle dependence. For the numerical analysis we will only consider the distinct cases I, IIa, and IIb $(\alpha=\pi / 4)$. We have taken $x$ in the range $[0.1,0.82]$ and have a cut on the polar angle in the $e^{+} e^{-}$c.m.s. lab frame, $\left|\cos \theta_{\text {lab }}\right|<0.95$. The results are presented for $\sqrt{s}$ in the range $[500,1500] \mathrm{GeV}$. Further the various polarization states are considered denoted by $\left(P_{e 1}, P_{l 1}, P_{e 2}\right)$ where $P_{e 1}$ and $P_{l 1}$ corresponds to the parent electron and laser beam and $P_{e 2}$ the other electron beam which interacts with the photon beam in the subprocess. The polarization state $(0,0,0)$ corresponds to the unpolarized case.

In Fig. 1 the various polarization states of case I has been plotted as a function of $\sqrt{s}$ in the range $[500,1500] \mathrm{GeV}$ for $\Lambda_{\mathrm{NC}}=500 \mathrm{GeV}$. The polarization state $(+,+,+)$ is the most dominant. To compare this with the commuting limit (SM), we have plotted the unpolarized cross section $(0,0,0)$ for $\mathrm{NC}$ and $\mathrm{SM}$ for case I with $\Lambda_{\mathrm{NC}}=500 \mathrm{GeV}$. The SM contribution drops much faster than the NC contribution at larger $\sqrt{s}$ and hence can be probed at the NLC. This behavior is typical for each of the NC cases under consideration and as the NC scale $\Lambda_{\mathrm{NC}}$ is increased the drop with $\sqrt{s}$ becomes

TABLE I. 95\% C.L. limits on the NC scale $\Lambda_{\mathrm{NC}}$ in $\mathrm{GeV}$ for $\sqrt{s}=500,1000,1500 \mathrm{GeV}$ obtained using an integrated luminosity $\int L=500 \mathrm{fb}^{-1}$. The various polarization states that yield the limits is indicated as $\left(P_{e 1}, P_{l 1}, P_{e 2}\right)$.

\begin{tabular}{lccc}
\hline \hline NC & $\sqrt{s}=500$ & $\sqrt{s}=1000$ & $\sqrt{s}=1500$ \\
\hline Case I & $1050(+-+)-1190(+++)$ & $1780(+--)-1840(+-+)$ & $2410(+++)-2530(+-+)$ \\
Case IIa & $890(++-)-1140(+++)$ & $1530(++-)-1730(+++)$ & $2080(++-)-2340(+-+)$ \\
Case IIb & $990(++-)-1150(+++)$ & $1670(+--)-1780(+++)$ & $2270(+--)-2440(+-+)$ \\
\hline \hline
\end{tabular}




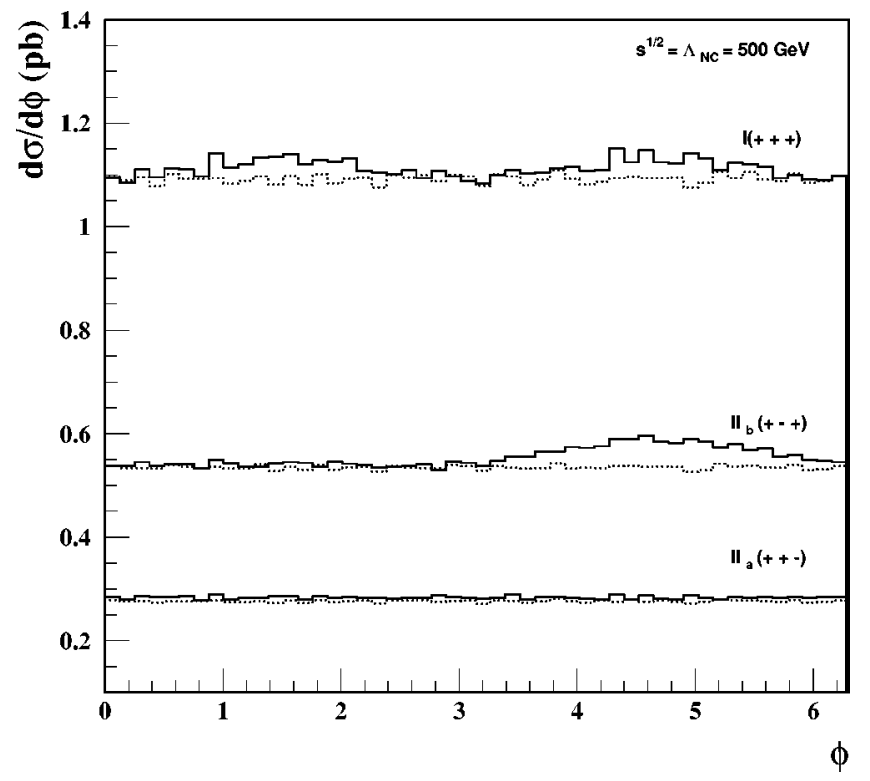

FIG. 2. Azimuthal angle dependence for various case of noncommutativity and polarization. The solid line denotes NC and the dotted line denotes the corresponding SM.

similar to the SM case though it is still higher. To study the reach possibilities at NLC as a function of the NC scale $\Lambda_{\mathrm{NC}}$ we perform a $\chi^{2}\left(\Lambda_{\mathrm{NC}}\right)$ fit using

$$
\chi^{2}\left(\Lambda_{\mathrm{NC}}\right)=\frac{L}{\sigma_{\mathrm{SM}}}\left[\sigma_{\mathrm{SM}}-\sigma_{\mathrm{NC}}\left(\Lambda_{\mathrm{NC}}\right)\right]^{2},
$$

where $L$ is the luminosity, $\sigma_{\mathrm{SM}}$ is the SM total cross section, and $\sigma_{\mathrm{NC}}$ is the NC QED total cross section. We choose an integrated luminosity $\int L=500 \mathrm{fb}^{-1}$ for the calculation. We obtain the $95 \%$ C.L. lower bound on $\Lambda_{\mathrm{NC}}$ by demanding $\chi^{2}\left(\Lambda_{\mathrm{NC}}\right) \geqslant 4$. The reach at NLC for various NC cases have

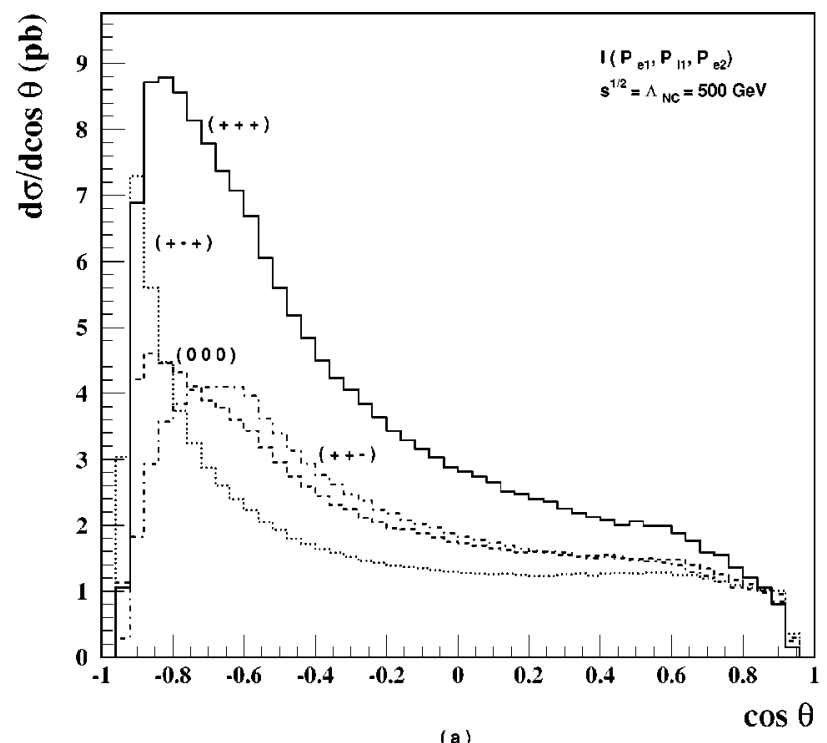

(a)

FIG. 3. (a) The differential cross section for noncommutativity case I as a function of $e \gamma$ c.m.s. polar angle $\cos \theta$ for $\sqrt{s}=\Lambda_{\mathrm{NC}}$ $=500 \mathrm{GeV}$ for various polarization states. (b) The differential cross section for the polarization state $(+,+,-)$ for NC and SM. been tabulated in Table I for $\sqrt{s}=500,1000$, and $1500 \mathrm{GeV}$. The various polarization states give different search limits and the range has been summarized in Table I.

The the typical azimuthal angle dependence of the various $\mathrm{NC}$ cases have been plotted in Fig. 2, for $\sqrt{s}=\Lambda_{\mathrm{NC}}$ $=500 \mathrm{GeV}$. Inspection of Eq. (16) shows the $\phi$ dependence, which corresponds to the choice of noncommutativity. Case IIa is independent of $\phi$ while cases I and IIb have distinct $\phi$ dependence as shown in Fig. 2.

The polar angle dependence of the SM and NC is different as can be see in Eq. (11). The SM has $1 / u$ dependence and hence will peak in the backward direction while the NC case has an additional $1 / t^{2}$ dependence and hence also has a forward dominance. The NC cross section has been plotted as a function of $e \gamma$ c.m.s. polar angle $\cos \theta$ for $\sqrt{s}=\Lambda_{N C}$ $=500 \mathrm{GeV}$. In Fig. 3(a) the various polarization states have been compared. To exhibit the forward dominance of NC case we have plotted in Fig. 3(b) the $(+,+,-)$ polarization state in comparison to the SM.

\section{CONCLUSIONS}

In this paper we have considered the Compton scattering in the noncommutative QED and studied the various distributions at the NLC in the $e \gamma$ mode. The NC phase factor $\Delta_{C}$ has contributions from space-space and space-time noncommutativity. We have considered the two cases separately. The various noncommutative cases considered have distinct azimuthal angle dependence. The polar angle dependence for noncommuting and the commuting SM is also different and can be used to probe the noncommutativity. The 95\% C.L. reach possibilities of NC scale $\Lambda_{\mathrm{NC}}$ for various noncommuting cases at the NLC operating in the $\sqrt{s}$ range $[500,1500]$ $\mathrm{GeV}$ has been given in Table I.

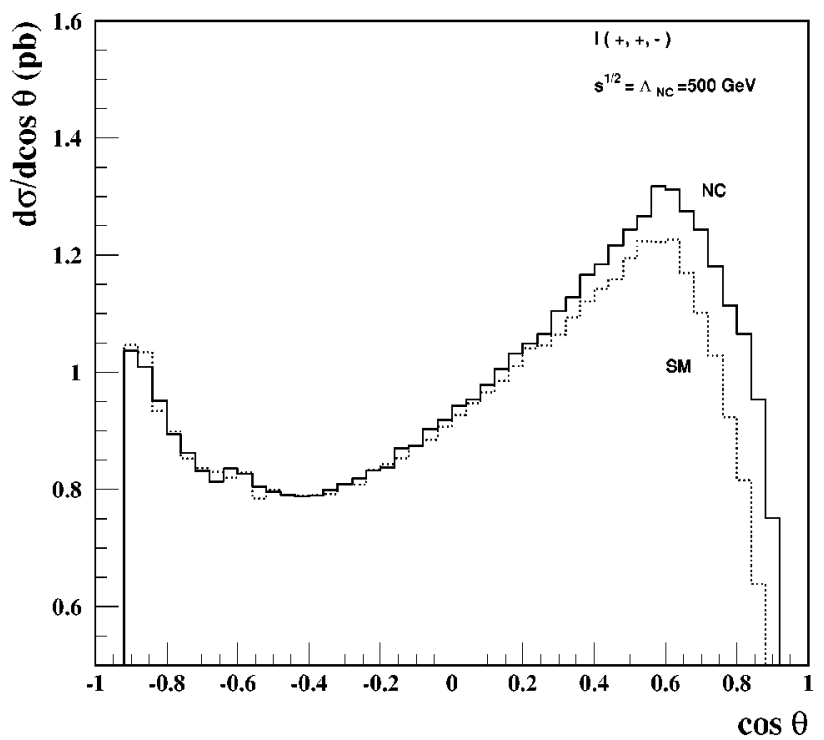

(b) 


\section{ACKNOWLEDGMENTS}

I would like to thank Keshav Dasgupta and Oscar J. P. Eboli for useful discussions. I also thank Oscar for going through the manuscript and for useful comments. Thanks are also due to Adi Armoni for his comments on the manuscript. This work was supported by FAPESP (Processo: 99/053109).

\section{APPENDIX}

This appendix contains the useful formulas for the distribution of photon produced using the laser back scattering technique. The electron beam responsible for the production of photon beam is called the parent electron. For further details regarding laser back scattering technique, refer to the original work [19]. The photon beam produced by laser back scattering has number density given by the distribution

$$
f\left(x, P_{e}, P_{l} ; z\right)=\frac{2 \pi \alpha^{2}}{m_{e}^{2} z \sigma_{C}} C(x),
$$

where $P_{e}$ is the polarization of the electron beam and is taken at $90 \%$ level $\left(P_{e}= \pm 0.9\right), P_{l}$ is the laser beam polarization and can achieve almost $100 \%\left(P_{l}= \pm 1\right)$. The fraction of the parent electron beam energy carried by the photon is denoted by $x$. The maximum value $x$ can take is 0.82 , above this value the laser beam and back scattered photon can pair produce $e^{+} e^{-}$pairs and hence decreases the efficient of the process. $x_{\max }=z /(1+z)$ and the optimal value for $z$ is $z_{\mathrm{opt}}=2(1+\sqrt{2})$. The function $C(x)$ and $\sigma_{C}$ is given by

$$
\begin{aligned}
C(x)= & \frac{1}{1-x}+1-x-4 r(1-r)-P_{e} P_{l} r z(2 r-1)(2-x), \\
\sigma_{C}= & \frac{2 \pi \alpha^{2}}{m_{e}^{2} z}\left\{\left(1-\frac{4}{z}-\frac{8}{z^{2}}\right) \ln (1+z)+\frac{1}{2}+\frac{8}{z}\right. \\
& -\frac{1}{2(1+z)^{2}}+P_{e} P_{l}\left[\left(1+\frac{2}{z}\right) \ln (1+z)-\frac{5}{2}\right. \\
& \left.\left.+\frac{1}{1+z}-\frac{1}{2(1+z)^{2}}\right]\right\}
\end{aligned}
$$

where $r=x /(z(1-x))$. The Stokes parameter is defined as

$$
\begin{aligned}
\xi_{2}\left(x, P_{e}, P_{l} ; z\right)= & \frac{P_{e}}{C(x)}\left(\frac{x}{1-x}+x(2 r-1)^{2}\right) \\
& -\frac{P_{l}}{C(x)}(2 r-1)\left(1-x+\frac{1}{1-x}\right) .
\end{aligned}
$$

[1] H. S. Snyder, Phys. Rev. 71, 38 (1947); 72, 68 (1947).

[2] A. Connes, M. R. Douglas, and A. Schwarz, J. High Energy Phys. 02, 003 (1998); M. R. Douglas and C. Hull, ibid. 02, 008 (1998); V. Schomerus, ibid. 06, 030 (1999); N. Seiberg and E. Witten, ibid. 09, 032 (1999).

[3] A. Connes, Non-commutative Geometry (Academic, New York, 1994).

[4] T. Filk, Phys. Lett. B 376, 53 (1996); J. C. Varilly and J. M. Gracia-Bondia, Int. J. Mod. Phys. A 14, 1305 (1999); M. Chaichian, A. Demichev, and P. Presnajder, Nucl. Phys. B567, 360 (2000); J. Math. Phys. 41, 1647 (2000); M. M. Sheikh-Jabbari, J. High Energy Phys. 06, 015 (1999); C. P. Martin, D. Sanchez-Ruiz, Phys. Rev. Lett. 83, 476 (1999); T. Krajewski and R. Wulkenhaar, Int. J. Mod. Phys. A 15, 1011 (2000); S. Cho, R. Hinterding, J. Madore, and H. Steinacker, Int. J. Mod. Phys. D 9, 161 (2000); E. Hawkins, hep-th/9908052; D. Bigatti and L. Susskind, Phys. Rev. D 62, 066004 (2000); N. Ishibashi, S. Iso, H. Kawai, and Y. Kitazawa, Nucl. Phys. B573, 373 (2000); I. Chepelev and R. Roiban, J. High Energy Phys. 05, 037 (2000); H. Benaoum, Nucl. Phys. B585, 554 (2000); A. Armoni, ibid. B593, 229 (2001).

[5] S. Minwalla, M. Van Raamsdonk, and N. Seiberg, hep-th/9912072.

[6] N. Seiberg, L. Susskind, and Nicolaos, J. High Energy Phys. 06, 004 (2000).

[7] R.-G. Cai and N. Ohta, J. High Energy Phys. 10, 036 (2000).
[8] C. P. Martin and D. Sanchez-Ruiz, Phys. Rev. Lett. 83, 476 (1999).

[9] M. Hayakawa, Phys. Lett. B 478, 394 (2000); hep-th/9912167.

[10] M. M. Sheikh-Jabbari, Phys. Rev. Lett. 84, 5265 (2000).

[11] K. Morita, hep-th/0011080.

[12] J. L. Hewett, F. J. Petriello, and T. G. Rizzo, hep-ph/0010354.

[13] I. Mocioiu, M. Pospelov, and R. Roiban, Phys. Lett. B 489, 390 (2000)

[14] I. F. Riad and M. M. Sheikh-Jabbari, J. High Energy Phys. 08, 045 (2000); N. Chair and M. M. Sheikh-Jabbari, hep-th/0009037.

[15] M. Chaichian, M. M. Sheikh-Jabbari, and A. Tureanu, hep-th/0010175.

[16] H. D. Abarbanel and M. L. Goldberger, Phys. Rev. 165, 1594 (1968).

[17] N. Arkani-Hamed, S. Dimopoulos, and G. Dvali, Phys. Lett. B 429, 263 (1998); Phys. Rev. D 59, 086004 (1999); I. Antoniadis, N. Arkani-Hamed, S. Dimopoulos, and G. Dvali, Phys. Lett. B 436, 257 (1998).

[18] L. Randall and R. Sundrum, Phys. Rev. Lett. 83, 3370 (1999); 83, 4690 (1999).

[19] I. F. Ginzburg, G. L. Kotkin, V. G. Serbo, and V. I. Telnov, Nucl. Instrum. Methods 205, 47 (1983); I. F. Ginzburg, G. L. Kotkin, S. L. Panfil, V. G. Serbo, and V. I. Telnov, ibid. 219, 5 (1984); V. I. Telnov, Nucl. Instrum. Methods Phys. Res. A 294, 72 (1990). 